\title{
Counting atoms
}

\author{
Enrico Massa and Giovanni Mana expound on the substance of the Avogadro constant.
}

$\mathrm{T}$ he need for a physical constant quantifying a reference amount of substance is a manifestation of the atomic nature of matter. At the basis of the concept of a 'quantity constant' lies the law of constant proportions: a pure compound always contains the same elements the conserved quantities in chemical reactions - in the same mass ratio.

This law played a fundamental part in the metrological development of chemistry: the amount of a given element or compound can be traced back to the amount of substance in $12 \mathrm{~g}$ of ${ }^{12} \mathrm{C}-\mathrm{a}$ mole of ${ }^{12} \mathrm{C}-$ by chains of stoichiometric proportions. For example, because $12 \mathrm{~g}$ of ${ }^{12} \mathrm{C}$ reacts with $4 \mathrm{~g}$ of $\mathrm{H}_{2}$ (approximately, ignoring less abundant isotopes) and $\mathrm{C}+2 \mathrm{H}_{2} \rightarrow \mathrm{CH}_{4}$, one mole of molecular hydrogen corresponds to $2 \mathrm{~g}$. Next, as $2 \mathrm{~g}$ of $\mathrm{H}_{2}$ reacts with $16 \mathrm{~g}$ of $\mathrm{O}_{2}$ and $\mathrm{O}_{2}+2 \mathrm{H}_{2} \rightarrow$ $2 \mathrm{H}_{2} \mathrm{O}$, the molar mass of molecular oxygen is $32 \mathrm{~g}$.

In the nineteenth century, investigations of a number of chemists brought to light clues that 'amount of substance' is discrete: it always comes in integer quanta; basic amounts that take part in chemical reactions. These 'basic amounts' were found to be constant independent of time, place or temperature. This strengthened the atomic hypothesis and linked the vindication of the existence of atoms to determining the number of quanta in a mole, the Avogadro constant $N_{\mathrm{A}}$, introduced in 1909 by Jean Perrin and named after Amedeo Avogadro, who pioneered the idea of molecules.

The original reference substance was chosen to be hydrogen - in modern parlance, the mole was set to the number of atoms in $1 \mathrm{~g}$ of $\mathrm{H}$. Later, oxygen was used, because it forms more compounds. Eventually, in $1960,{ }^{12} \mathrm{C}$ was chosen.
Although the concept of the atom dates back to the Greek philosopher Democritus, there was no experimental proof of atomism until the end of the nineteenth century. One remarkable attempt to count the atoms in a finite amount of substance was made by Johann Magnenus, who reported in 1646 that the scent of burned incense fills a church of more than seven hundred million times its original size and that this observation can be used to arrive at a primitive estimate of an atom's size ${ }^{1}$. From the ratio of the volumes of the interior of the church and the minimal amount of evenly dispersed incense that one can sense, which he estimated to be to one-thousandth of a chickpea and guessed to contain at least a million atoms, Magnenus arrived at $7.776 \times 10^{17}$ as a lower bound to the number of atoms in an incense chickpea. What he did - although not very reliably — is essentially the same thing we do these days to count atoms in silicon crystals. Both approaches ingeniously amplify the atomic scale so that macro-scale detection is possible: Magnenus used diffusion and smelling, we use crystallization and X-ray diffraction. From 1865 to 1965 more than eighty determinations of $N_{\mathrm{A}}$ were reported, obtained by means of more than twenty different methods ${ }^{2}$; notable cases include those by Loschmidt (kinetic theory of gases), Perrin (Brownian motion) and Einstein (diffusion of particles in a fluid).

Until 1965, when Bonse and Hart demonstrated the first X-ray interferometer ${ }^{3}$, the value of $N_{\mathrm{A}}$ was used for determining the lattice spacings in crystals from their volumes and masses. Now, X-ray interferometry enables counting how many silicon atoms are aligned in a crystal, the length of which is measured by optical interferometry, relating atomic dimensions to the wavelength of visible light ${ }^{4}$. Using this method, the number of atoms in a $1 \mathrm{~kg}$ sphere of high-purity ${ }^{28} \mathrm{Si}$ (an artist's impression is pictured, featuring Avogadro and his law) was counted and $N_{\mathrm{A}}$ determined by dividing the molar volume by the atomic volume of ${ }^{28} \mathrm{Si}$ measured via X-ray interferometry. The current most accurately measured value of $N_{\mathrm{A}}, 6.02214076 \times 10^{23} \mathrm{~mol}^{-1}$ with a relative standard uncertainty of 20 parts in $10^{9}$, has been obtained in this way by the International Avogadro Coordination project ${ }^{5}$.

High-precision measurements of the Avogadro constant are important for being able to quantitatively connect the macroand microscales - obviously, knowing $N_{\mathrm{A}}$, one can switch between molar mass and particle mass. But in addition, metrologists are planning to define the kilogram and the mole via the Planck and Avogadro constants ${ }^{6}$. Before that can happen, an accurate determination of $N_{\mathrm{A}}$ needs to be realized. By fixing $N_{\mathrm{A}}$, the atomic model of matter becomes embedded in the International System of Units (SI) and the mole redefined in terms of the number of entities it represents. Furthermore, as the ratio between the ${ }^{28} \mathrm{Si}$ mass and the Planck constant can be accurately measured using atom interferometry, and as we are now able to 'count atoms', macroscopic prototypes with an exactly known amount of substance and mass can be realized (neglecting Einstein's mass-energy equivalence principle and atoms therefore having unique and well-defined masses).

ENRICO MASSA and GIOVANNI MANA are at the Istituto Nazionale di Ricerca Metrologica, Strada delle Cacce 91, 10135 Torino, Italy.

e-mail:e.massa@inrim.it; g.mana@inrim.it

References

1. Magnenus, J. C. in Democritus Reviviscens 207 (Wyngaerden, 1646).

2. Mana, G. \& Zosi, G. Rivista del Nuovo Cimento 18, 1-23 (1995)

3. Bonse, U. \& Hart, M. Appl. Phys. Lett. 6, 155-156 (1968).

4. Massa, E. et al. J. Phys. Chem. Ref. Data 44, 031208 (2015).

5. Azuma, Y. et al. Metrologia 52, 360-375 (2015).

6. Fischer, J. \& Ullrich, J. Nature Phys. 12, 4-7 (2016).

$\mathrm{m} e \AA \mathrm{s} \mathrm{u} R E_{\mathrm{h}}$

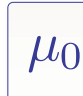

$\varepsilon_{0}$

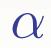

$\sigma$

